\title{
Filosofía de la educación para la primera infancia*
}

\section{Philosophy of early childhood education}

«Centrarse en los niños pequeños no es un lujo, ni una opción, es el núcleo donde se fortalecen los círculos que conforman una sociedad y determinan los cotextos de todos los problemas sociales»

(www.redprimerainfancia.org)

Romelia Negret Doria**

ReCIBIDO: 3 DE FEBRERo de 2012 • APRobAdo: 17 DE OCTUBRE DE 2012

Resumen. En el presente artículo se exponen algunas ideas sobre la educación para la primera infancia, los postulados que guían la educación en esta etapa y la importancia de comprender lo decisivo que resultan estos primeros años en la vida de los niños y niñas para su aprendizaje y desarrollo en el presente y futuro de una sociedad, al considerar todas las dimensiones humanas.

Palabras clave. Infancia, educación, humanización, valores, comprensión, cerebro.

* Artículo de reflexión que indaga sobre la filosofía de la educación para la primera infancia, en desarrollo de la Línea de Investigación Educación y Políticas Públicas, Doctorado en Educación en la Atlantic International University (AIU).

** Licenciada en Ciencias Sociales de la Universidad de Córdoba, con estudios de Posgrado en Enseñanza de la Historia de la Pontifica Universidad Javeriana de Bogotá y Maestría en Sociología de la Educación de la Universidad Pedagógica Nacional. Desde hace varios años se viene desempeñando como docente en el área de las Ciencias Sociales y la Filosofía en la Secretaría de Educación de Bogotá. Ha sido asesora pedagógica en varios colegios, participante de talleres y cursos para formación de maestros en convenios con FUNDACIES-MEN, tutora on line para América Latina con la Escuela de Graduados en Educación (Proyecto Cero) de la Universidad de Harvard y en la actualidad candidata a doctorado en educación en la Atlantic International University (AIU), Estados Unidos. E-mail: rnegretedoria@yahoo.com 


\begin{abstract}
This paper presents shows some ideas about early childhood education, the principles that guide education at this stage and the importance of understanding what are these first crucial years in the life of children for learning and development in the present and future of a society, to consider all human dimensions.
\end{abstract}

Keywords. Childhood, education, humanization, values, understand, brain.

\title{
Introducción
}

¿Qué postulado de la educación puede ser concebido y diseñado dejando por fuera los decisivos primeros años de infancia? Cada vez más los estudios provenientes de diferentes disciplinas demuestran que estos primeros años de vida son los fundamentales para el desarrollo emocional, social, físico y cognitivo de todos los seres humanos. ¿Qué pedagogía se hace necesaria para favorecer estos desarrollos? Ya algunos investigadores en pedagogía como Romero (2008) afirman que «hablar de una pedagogía de la humanización en la edad inicial es hablar de comprensión, convivencia, tolerancia, responsabilidad social, amor, interlocución, respeto, valoración de si y del otro».

Frente a lo anterior existe una preocupación por parte de varios agentes sociales, gubernamentales y no gubernamentales interesados en aportar a crear no solo legislaciones, sino informes a favor de la primera infancia donde se presentan otras perspectivas de educación pensada para estas primeras edades. En especial se recalcan los tres primeros años de vida de los niños en los que el cerebro logra un mayor desarrollo en completa dependencia de sus genes, pero también de su entorno, nutrición, salud e interacciones humanas que percibe y recibe en su círculo familiar y social.

¿Qué ocurre en los sitios dedicados a prestarle atención a estos primeros años? ¿Qué responsabilidad tienen los encargados de desarrollar no solo habilidades y destrezas sino funciones de pensamiento social, emocional entre otros? Ya que igualmente se desarrollan las habilidades para pensar, hablar, aprender y razonar con un gran impacto sobre el aprendizaje presente y posterior de niños y niñas. Lo anterior surge debido a las condiciones en que reciben educación los niños y niñas pertenecientes a estratos socio económicos poco favorables en Colom- 
bia y que, en ocasiones, reciben lo que medianamente pueden ofrecer personas encargadas más de su cuidado que de su desarrollo cognitivo, social y emocional.

Pero cómo pueden los niños en estos primeros ciclos de vida tener atención y una educación donde los procesos emocionales, físicos, sociales y cognitivos se desenvuelvan apropiadamente y contribuyan a ampliar sus oportunidades a lo largo de sus vidas, enmarcado en una educación de calidad en la que se valoren, desde la sociedad, otras formas de educar sin temor, violencia o amenaza, ya que la escuela por su tradición de trasmisora de cultura sigue haciendo énfasis más en lo cognitivo que en lo emocional (Romero, 2008). Organismos internacionales, como la UNICEF (2001), han mencionado que «la atención, cuidado y educación a la primera infancia es concebida como una estrategia efectiva para reducir la inequidad», pero los resultados de los informes en Colombia sobre los niveles de pobreza e inequidad en algunas poblaciones parecen contradecir este propósito; cada vez son más los niños y niñas en edad escolar que están por fuera del sistema de cobertura y que demuestran que cerrar la brecha en este nivel es una tarea compleja. Aun cuando se argumente que los aprendizajes significativos en estos primeros años favorecen el desarrollo humano al contribuir indirectamente con el desarrollo sostenible, el logro de la paz y la estabilidad de los pueblos, en ocasiones la realidad parece negar esto.

Existe una gama de argumentos por los cuales la atención a la primera infancia es importante; entre los presupuestos están, como se mencionó anteriormente, en primer lugar, que es la etapa fundamental de desarrollo; luego que esta impacta positivamente el proceso social y cultural de un pueblo porque disminuye la desigualdad social, porque mejora el acceso y permanencia en el sistema escolar y porque es rentable económicamente. Veamos qué hay detrás de cada uno de ellos.

Las investigaciones realizadas en el campo de la neurociencia, ciencias del comportamiento, psicología del desarrollo, biología, educación y economía han arrojado que desde la etapa de gestación los infantes cuentan con capacidades físicas, cognitivas, sociales y emocionales que se deben reconocer y promover pues son la base para el aprendizaje, la comunicación, la socialización y, en general, para el desarrollo de habilidades, capacidades y competencias. Según Ratey (2002) cuando el niño nace tiene millones de conexiones buenas que esperan una asignación 
concreta, un trabajo para hacer esto sucede a medida que el mundo y el entorno lo exige, así se va requiriendo conexiones neuronales para tareas concretas como ver, recordar, hablar, jugar, escribir, etc. Según las investigaciones hechas por el científico las conexiones que no se usan o estimulan en los primeros años de vida acaban por ser eliminadas (0 a 6 años). A falta de una estimulación adecuada una célula cerebral morirá, pero si se le estimula y alimenta con experiencias enriquecidas brotarán nuevas conexiones y ramas de conexiones neuronales. Justo en los primeros años de vida, las conexiones de las neuronas que sobreviven y son estimuladas se comunican con la rapidez del fuego en la sinapsis y cuanto más se utilice una determinada conexión más fuerte será esa ruta. Es por lo anterior que la enseñanza en esta etapa debe tener presente toda esta actividad cognitiva que los niños y niñas realizan antes de enfrentarse a las clases en la escuela para continuar fortaleciéndola y es el proceso de lectura por ejemplo donde se evidencia este aspecto, tal como afirma Guzmán (2009).

Ya la UNICEF (2006) afirmaba que:

La nutrición en la primera infancia junto con la estimulación temprana son factores determinantes de las funciones neurológicas y favorecen los aprendizajes, la salud y un comportamiento satisfactorio a lo largo de la vida, donde la leche materna juega un papel esencial en el desarrollo del cerebro, pues nacemos con más de 100 billones de neuronas, pero que un 95\% no están conectadas entre sí, es solo hasta el nacimiento que se inicia esta interconexión que permite ,ver, escuchar, hablar, pensar, sentir, etc. Durante la etapa de lactancia se produce la melanina sustancia grasa de la leche materna, que facilita la conducción de las vías neuronales necesaria para el desarrollo del cerebro, que hacia los tres años se darán en abundancia como sinapsis.

Otros circuitos son los sensoriales, básicos para el desarrollo cognitivo y del lenguaje e importante para estímulos emocionales, el sistema de inmunización y el sistema nervioso central. Es en estos donde el medio sociocultural y económico afecta el desarrollo según Mustarad (2002) pero, si llega a producirse el maltrato se altera el circuito de producción de serotonina con consecuencias de aumento de estrés que en la edad adulta se reflejará en los comportamientos sociales alterados y que justifican una política pública dirigida a prevenir que el maltrato aumente durante estos primeros años. El autor concluye diciendo que «el estimulo 
externo e interno, que se recibe durante el periodo de gestación y posterior al nacimiento es determinante para el desarrollo posterior». Existen evidencias afirma la UNICEF (2006) de como el abandono y la pobreza durante los primeros años afecta estructuras química del cerebro y su organización, igual que la vinculación afectiva con los padres afecta positivamente el desarrollo humano y que se asocia a mejores logros académicos y sociales y por consiguiente a un mejor desempeño escolar, como lo demuestran otras investigaciones al respecto.

Otra razón de las políticas encaminadas a la primera infancia se refiere a que mejora el acceso y permanencia en el sistema educativo, plantea una comparación entre países desarrollados como en vía de desarrollo sobre porcentajes altos (95\%) que demuestra que estudiantes que ingresaron a la educación en la primera infancia e ingresaron después a la primaria en comparación a otros (75\%) que no tuvieron estos programas. Es por supuesto una conclusión clara que los resultados de un ingreso a temprana edad a programas escolares favorece la adaptabilidad social, el desarrollo cognitivo y social de los niños y niñas. Pero en este punto solo se limita a mostrar la comparación entre los que tienen acceso a la educación y no se enfoca a una revisión de las mismas políticas frente a los que siguen sin acceder a la escolaridad temprana, será que los informes solo muestran una cara de la realidad para seguir empeñados en mostrar la pertinencia de los programas aunque los resultados no sean totalmente amplios ni satisfactorios? ¿Cómo impactan los procesos sociales y culturales la atención en la primera infancia?

Cada vez más es evidente la transformación familiar y como repercute en el cuidado y protección de los niños y niñas en edad escolar, la mujer en el campo laboral como cabeza de hogar, los factores de desplazamiento, violencia intrafamiliar han sido elementos que ameritan que la política de primera infancia ocupe un lugar importante en la sociedad. Cada vez más hay agentes que intervienen en la crianza de los hijos, donde la ausencia de los padres favorece que parientes cercanos apoyen el cuidado e incidan en forma indistinta en la formación de valores, principios y desarrollo de habilidades cognitivas y sociales en general, que asociados a el maltrato, abandono y desvinculación afectiva van a influir en su desarrollo infantil no tan positivamente, sino por el contrario frenando sus capacidades y posibilidades de desarrollo cognitivo, emocional y social, etc. La violencia social que padecen familias por el desplazamiento forzado ha influido en el desarrollo normal de niños y 
niñas que en varias ocasiones, como afirman los informes CODHES \& OIM (2003), demuestran que la situación en Colombia es dramática para los infantes que han llegado a ser reclutados o se ven obligados a trabajar en detrimento de su desarrollo emocional, cognitivo, social y emocional. ¿Qué costos tiene para una sociedad que sus infantes vayan a la guerra primero que a la escolaridad y que además sean separados de su núcleo familiar básico? Y, además, ¿̇cómo conservar las tradiciones y costumbres culturales cuando son los infantes los primeros separados de su familia y comunidad cercana? ¿Quién puede garantizar esto? Si la preocupación es la simple supervivencia, pues fuera de la familia no queda otra cosa que enfrentar la realidad de estar en otros grupos no por cierto empeñados en su formación social y cultural.

¿En qué momento se inicia el encuentro entre textos, lenguaje y cultura? No es acaso en estos primeros años de infancia, cuando la fantasía está suelta, la imaginación vuela y los diálogos interculturales florecen en diferentes escenarios como el familiar, el escolar, los amigos y la comunidad. Hay voces, movimientos, gestos y aprendizajes rondando alrededor de los niños y niñas que dicen qué cultura viven qué deseos pueden realizar y qué sociedad quieren transformar. Es la construcción de la vida social y cultural que en los primeros años de vida disfrutan los infantes. Ya lo menciona Pérez (2006) es la oralidad y la lectura que favorece la familia y la institución la que conjuga el mundo de los primeros años de niños y niñas, todos tienen derechos a participar de la vida cultural y a formar su valoración estética sobre la literatura, la música, las artes en general. Por lo tanto, las políticas para la primera infancia deben garantizar que la interculturalidad y la diversidad de culturas en los grupos sean patrimonio de la primera infancia y que sus prácticas se preserven de generación en generación para mantener la cohesión cultural y social que toda comunidad necesita para hablar de identidad histórica.

Como vemos, la importancia de la educación en la primera infancia es un asunto de primer orden y llega hasta el plano de lo económico, según lo enunciado por los profesores Heckman y Carneiro, premios Nobel en economía en el 2000, «las inversiones a temprana edad tienen mayores tasas de retorno al capital humano, que las remediales en otros momentos de la vida. Pero, a la par con lo anterior, se encuentra la disminución de la desigualdad social; al haber mayor inversión en la población inicial hay mejores posibilidades de inserción laboral al mediano y largo plazo, 
aunque la tendencia mundial demuestre que cada vez la inversión es menor para los sectores de bajos recursos, donde los costos de inversión van a la par de la baja calidad de la educación para los más pobres en comparación con los niños de capas medias y altas quienes reciben una educación mejor. El impacto es negativo, analizado desde esta perspectiva, y Colombia no está lejos de este parámetro ya que los programas en este sentido se han dirigido a programas de nutrición, salud y protección y es aún débil el componente educativo.

Sin embargo, en el marco de la política educativa de la llamada Revolución Educativa (2006-2010), la meta es brindar educación inicial complementada con nutrición y salud a 400.000 menores de 5 años, desde el 2006 hasta el 2010; tal como reza el documento oficial, se define muy bien los propósitos dentro de los derechos fundamentales y en el contexto familiar, comunitario e institucional.

Cuáles son los retos que enfrenta una política educativa de tan grande alcance: ampliar la cobertura con calidad, equidad, brindar a los padres de familia espacios donde sus hijos reciban atención y educación de calidad y, por último, fortalecer la atención en el ámbito educativo con el fin de realizar un tránsito apropiado entre la educación inicial y el ciclo de la básica primaria.

He venido usando el término infante, que proviene del latin infans, que significa mudo, que no habla, basado en creencias de que es el lenguaje el que convierte a los niños en seres comunicativos tal como lo describe Owens (2006). Pero desde la visión contemporánea de la antropología, la infancia resulta de la evolución reciente de la historia de la humanidad, ya que en los primeros tiempos no existía la infancia como etapa de desarrollo sino que una vez el niño caminaba era incorporado a la tarea de la supervivencia y a la recolección de productos para el grupo al que pertenecía. Esto solo va a cambiar con el correr de los años y los avances e investigaciones en el campo de la educación, psicología del desarrollo y otras disciplinas.

Pero son los padres y madres de familia los agentes educativos más influyentes en el desarrollo de la personalidad de los niños en sus primeros años, de modo que el entorno familiar se convierte en la etapa de la socialización primaria que lo prepara con valores y normas para enfrentar la segunda etapa la socialización secundaria, donde el entorno social, 
la educación escolar, la relación con los demás y el espacio geográfico y cultural son altamente importantes para continuar con su desarrollo.

Pero han sido los estudios sobre el desarrollo en esta etapa, los que han contribuido a su mayor comprensión y sobre todo a ser incluidas en políticas educativas, a través de programas orientados a favorecer el desarrollo de los niños y niñas en todas las dimensiones humanas. Las teorías piagetianas pusieron mucho énfasis en la evolución y el desarrollo y escasa atención a la parte de la socialización, así como la tesis de Chomsky sobre el desarrollo del lenguaje que presta poca atención a los aspectos socio pragmáticos de las interacciones sociales. Solo hasta los años setenta se produjo un interesante cambio de orientación y fue la teoría de Vygotsky, corriente interaccionista, en la que se plantea que son las variables sociales las fundamentales para el desarrollo y donde la resciliencia juega un papel importante en la socialización pues se evidencia en la relación persona y entorno y que el ser humano es capaz de sobre ponerse a situaciones de vulnerabilidad.

Pero cómo encontrar un equilibrio entre la naturaleza y la crianza y dar el estímulo adecuado en la primera infancia con el ánimo de garantizar un mejor nivel de aprendizaje, una mejor motricidad, memoria, un adecuado manejo de las emociones, un adecuado desarrollo del lenguaje, para que todo esto conduzca a un cerebro social que le permita a las personas enfrentar situaciones de manera positiva, ser creativos, mejorar los niveles de atención, etc., así muchos neurólogos y psicólogos han coincidido en reconocer que el comportamiento social es una función del cerebro exactamente igual que la memoria y el lenguaje. Ratey (2002) asegura que al unir las piezas de la naturaleza y el entorno se puede afirmar que en efecto existe un cerebro social. El autor menciona en su libro varios ejemplos para demostrarlo.

Así, considero que el aspecto neuropsicológico, social, biológico y educativo son elementos sustentadores de mucha importancia que vienen fortaleciendo el interés por las políticas de primera infancia y los programas de orientación y formación que de ésta surja.

Finalmente, son muchos los interrogantes que sobre el tema de la filosofía de la educación para la primera infancia se deben abodar, ya que como ha sido mencionado prestar atención a estos primeros años de vida es pensar cuál será la sociedad del futuro y en manos de quién está 
o sigue reposando esta responsabilidad. Vale la pena no dejar de lado este asunto ya que si bien ahora es una preocupación de los gobiernos no dejará de ser un tema de central investigación para la pedagogía, la psicología del desarrollo, la filosofía de la educación y las políticas de los Estados, entre otros.

A manera de cierre presento algunas inquietudes más que conclusiones sobre cómo la educación de la primera infancia debe ser el punto de partida en el diseño de políticas de calidad. ¿Cómo pensar en currículos educativos donde la primera infancia sea el punto de entrada y no de llegada? ¿Qué filosofía de la educación debe guiar los currículos para estos primeros años? ¿Cuál es el futuro de la educación para la primera infancia; un asunto político, social, pedagógico o de más agentes? Y, por supuesto, ¿de qué manera los derechos de los niños son abarcadores en estos primeros años? ¿Cómo desarrollar programas para el talento humano en estos años iniciales, más allá de las políticas públicas? El presente asunto plantea la necesidad de una educación consciente en estos primeros años y convoca a alternativas acordes con esta problemática. De ahí que proponer una educación humanizada desde la primera infancia hasta la etapa superior no puede estar desligada de su connotación vivencial, no puede ser meramente formal o estética. Este camino que muestra desde la filosofía de la educación una humanización es uno de los pasos más difíciles y complejos en la formación de seres humanos desde sus primeros años hasta la edad adulta, pero el reto se focaliza en la legislación, en las instituciones educativas, en los docentes, en las familias y, por supuesto, en la sociedad en su conjunto, siempre que la educación para estos primeros años sea pensada como un asunto de todos.

\section{Referencias}

CODHES \& OIM (2003). Destierros y desarraigos, memorias. Recuperado de http://www.cinde.org.co.

Guzmán, R. J. (2009). La planeación de las clases para la enseñanza de la lectura impacta la sociedad. En: Revista Internacional Magisterio, 37.

Ministerio de Educación Nacional (MEN). ¿̇Por qué una política educativa para la primera infancia?, Informe basado en El estado mundial de la infancia 2001, Primera infancia UNICEF. Recuperado de http:// www.colombiaaprende.edu.co 
(2009). Revolución Educativa: Plan Sectorial 2006-2010. Recuperado de http://www.colombiaaprende, edu.co

(2009). Argumentos científicos: es la etapa más importante para el ser humano. Recuperado de http://www.colombiaaprende.edu.co (2010). Educación de Calidad: Educación para la primera infancia. Recuperado de www. colombiaaprende.edu.co.

Mustarad (2002). Módulo 2 sobre desarrollo infantil. Recuperado de en http://primerainfancialac.org

Organización de las Nacionales Unidas (ONU). (2008). Objetivos de desarrollo del milenio. Recuperado de www.colombiaaprende.edu.co

Owens, C. (2006). Revolución educativa. Recuperado de http:// www. colombiaaprende.edu.co

Pérez, M. et ál. (2006). Acceso al lenguaje, la lectura y la escritura: Una prioridad de la Política de Infancia. Políticas de Primera infancia. Recuperado de http://www.cinde.org.co

Ratey, J. (2002). El cerebro manual de instrucciones. Barcelona: Editorial Arena Abierta.

Romero, P. (2008). Pedagogía de la Humanización en la educación inicial. Bogotá: Universidad San Buenaventura.

UNICEF, $(2001,2006)$. Revolución educativa. Recuperado de http:// www. colombiaaprende.edu.co y www.redprimerainfancia.org 